\section{RELATION BETWEEN STRUCTURAL PHASE TRANSITIONS AND TRANSFORMATION TWINS OF KLISO4}

Ch. Scherf ${ }^{1,2}$ N.R. Ivanov ${ }^{4}$ Th. Hahn ${ }^{2}$ H. Klapper ${ }^{3}$ G. Heger ${ }^{2}$

${ }^{1}$ Laboratoire Leon Brillouin, CEA Saclay, 91191 Gif-sur-Yvette, France

${ }^{2}$ Institut fuer Kristallographie, RWTH Aachen, 52056 Aachen, Germany

${ }^{3}$ Mineralogisch-Petrologisches Institut, Universitaet Bonn, Poppelsdorfer

Schloss, 53115 Bonn ${ }^{4}$ Institute of Crystallography, Russian Academy of

Sciences, 117333 Moscow, Russia

The tetrahedral framework structure of $\mathrm{KLiSO}_{4}$ exhibits a rich polymorphism between $5 \mathrm{~K}$ and $989 \mathrm{~K}$. The high-temperature phase transition cycles III-II-III and III-II-I-II-III (phase III: hexagonal polar $P 6_{3}$, phase II: orthorhombic Pcmn, phase I: hexagonal $\mathrm{PG}_{3} / \mathrm{mmc}$ ) are accompanied by the formation of three ferroelastic domain states in phase II (related by a treefold rotation around [001]) and at least two of the four possible domain states in phase III according to the three merohedral twin laws $m$ parallel [001], m normal [001] and 2 normal [001]. The domain structures were observed by polarization optics, liquid-crystal decoration and X-ray and synchrotron topography. Grown-in inversion twin lamellae of phase III vanish after the phase transition cycles. Only new m parallel [001] domains (same polarity) occur. This proves the disappearance of the polarity of phase III after the transformation into modification II. The structures of phase I $\left(\mathrm{Pb}_{3} / \mathrm{mmc}\right)$ and phase II $(\mathrm{Pcmn})$ are centrosymmetric and exhibit strong disorder of the tetrahedra in contrast to the ordered polar structure of phase III.

Keywords: PHASE TRANSITIONS TWINS POLARITY

Acta Cryst. (2002). A58 (Supplement), C250

\section{HOW ACCURATE ARE THE ELECTRON MOMENTUM AND CHARGE DENSITY MAPS OBTAINED BY THE MAXIMUM ENTROPY METHOD}

L. Dobrzynski $^{1,2}{ }^{\text {J. Waliszewski }}{ }^{1}$

${ }_{1}^{1}$ Institute of Experimental Physics, University of Bialystok, Lipowa Str.41, 15 424 Bialystok, Poland ${ }^{2}$ The Soltan Institute for Nuclear Studies, 05-400 Otwock-Swierk, Poland

One of the principal problems in reconstruction of the electron momentum and/or charge density maps by the Maximum Entropy Method consists in proper estimation of the corresponding error maps. In fact, this problem may be divided into several questions: how accurately the MEM equations are solved, what role is played by the chosen prior, are there any systematic errors imposed by the MEM algorithm as such, how well the Lagrange multiplier can be chosen within the framework of the Bayesian analysis etc. All these factors have influence on the quantitative analysis of error maps. We focus our attention on the reconstructions carried out mainly with the use of the MEEDtype algorithm and illustrate the problem by considerations of the error estimation in the one-dimensional case of the spherical electron momentum density and 3-dimensional problem of the charge density distributions in silicon and GaN. The natural limitations of the MEED are shown. The error maps are obtained by different approaches and compared with each other.

Keywords: MAXIMUM ENTROPY METHOD, CHARGE DENSITY DISTRIBUTION, COMPTON SCATTERING

\section{MULTIPOLE REFINEMENTS AND MAXIMISATION OF ENTROPY.} COMPETING OR COMPLEMENTARY METHODS

\section{N. K. Hansen}

Laboratoire de Cristallographie et Modelisation des Materiaux Mineraux et

Biologiques, UMR-CNRS-7036, Universite Henri Poincare - Nancy I, B.P.239, F-54506 Vandoeuvre les Nancy CEDEX, France

Detailed descriptions of the electron density distributions can be obtained from high-resolution X-ray diffraction experiments. The first studies were based on difference Fourier maps, but soon different approaches for modeling the fine details of the electron density were developed. Most successful, measured by the number of studies, are the so-called Multipole Models. Within the last decade, several electron density determinations have been made using Maximum Entropy Methods (MEM). We will attempt to make a formal description of the inversion problem, i.e. how to get from structure amplitudes to an electron density, which has the virtue of being able to describe both the MEM and the modeling (multipole or others) approach. A combination of the two would be very interesting for eliminating problems from which the methods taken individually suffers, which are: Multipole modeling: - the modeled electron density may not be overall positive - it is not evident, if the multipole model must/can describe all of the valence electrons MEM: - a socalled prior density must be given and the structure factor data must be phased - because of noise in the data, there is not a unique solution to the reconstruction problem - ambiguities due to prior density and the effect of random errors on the reconstructed density is difficult to analyze The strength of the multipole model approach is that the effects of thermal vibration are separated/deconvoluted from the density. Moreover the "multipole" program packages all contain powerful tools for analyzing electron densities and calculating properties.

\section{Keywords: ELECTRON DENSITY MULTIPOLE REFINEMENT} MAXIMUM ENTROPY METHOD

\section{Acta Cryst. (2002). A58 (Supplement), C250 \\ MEM CHARGE DENSITY STUDIES FOR MATERIALS WITH CHARACTERISTIC PHYSICAL PROPERTIES}

M. Sakata M. Takata E. Nishibori

Department of Applied Physics, Nagoya University, Nagoya, JAPAN

Since the advent of the third generation synchrotron radiation, it become much easier to determine accurate structures of materials, which posses interesting physical or other properties. In this context, the importance of the sophisticated analytical method, i.e. the Maximum Entropy Method, which can lead to have crystal structure at electron level even from powder specimen, seems to be increasing. In this talk, two kinds of aspects of the Maximum Entropy Method will be presented. One is how to improve structure model in MEM/Rietveld method, which is very powerful method to derive accurate charge densities from powder specimen. A few examples will be shown to demonstrate the usefulness of the method in materials science. The other is to discuss what can we do in materials science by obtaining the accurate charge density distributions (or crystalline structure at electron level). Are there any basic differences between structure determination at atomic level and structure refinements at electron level? If so, what is the main difference? A part of answers for these questions will be given in this talk from the viewpoint of materials scientists, who are interested in the relationships between structure and physical or other properties. In some cases, it seems un-doubtful that the crystal structure at electron level is far superior to that at atomic level for materials scientists to understand the above relationships.

Keywords: MAXIMUM ENTROPY METHOD,CHARGE DENSITY,SYNCHROTRON RADIATION 